Pesq. Vet. Bras. 35(2):148-152, fevereiro 2015

DOI: $10.1590 / \mathrm{S} 0100-736 \mathrm{X} 2015000200009$

\title{
Componentes principais das características de carcaças de bovinos anelorados e fontes de variação em lesões ${ }^{1}$
}

\author{
Fredson V. Silva ${ }^{2 *}$, Franklin D.S. Soares ${ }^{2}$, Laura L.S. Oliveira² ${ }^{2}$, Fábio L.B. Toral ${ }^{3}$, Sidnei \\ T. Reis ${ }^{2}$, Dorismar D. Alves², Vicente R. Rocha Júnior ${ }^{2}$ e Ricardo R.R. Gomes ${ }^{2}$
}

\begin{abstract}
Silva F.V., Soares F.D.S., Oliveira L.L.S., Toral F.L.B., Reis S.T., Alves D.D., Rocha Júnior V.R. \& Gomes R.R.R. 2015. [Principal components of carcass characteristics in Zebu cattle and variation factors for injuries.] Componentes principais das características de carcaças de bovinos anelorados e fontes de variação em lesões. Pesquisa Veterinária Brasileira 35(2):148-152. Departamento de Ciências Agrárias, Universidade Estadual de Montes Claros, Avenida Reinaldo Viana s/n, Janaúba, MG 39440-000, Brazil. E-mail: fredson.silva@unimontes.br

This study was made in order to evaluate the principal components of carcass characteristics in Zebu cattle and variation factors for injuries. We used a database with information from 15,002 carcasses of Zebu cattle. The variables studied were hot carcass weight, carcass conformation, fat thickness score, sexual condition, number of teeth, injuries and distance from the farm to the slaughterhouse. We also raised the finishing system of cattle through information obtained from the technician responsible for the herd. To understand the relationship of the variables, we used the correlation of the principal components and original variables, the factorial plans, the unit circle, cluster analysis and non-parametric tests. The fat thickness score, sexual condition, hot carcass weight, the number of teeth, and farm comprised $68.26 \%$ of the total variability. The carcasses conformation and the finishing system explained a low proportion of the variability. Variables as farm, number of teeth, finishing system and fat thickness score influenced the number of injuried carcasses. The sexual condition, hot carcass weight and carcass conformation did not change the proportion of injuried carcasses.
\end{abstract}

INDEX TERMS: Carcass classification, bulls, oxen, contusions, hematomas.

RESUMO-- Objetivou-se levantar e avaliar os componentes principais das características de carcaças de bovinos anelorados e fontes de variação em lesões. Utilizou-se um banco de dados com informações de 15.002 carcaças de bovinos anelorados. As variáveis levantadas foram peso da carcaça quente, conformação da carcaça, escore de gordura subcutânea, condição sexual, número de dentes incisivos, lesões e distância percorrida da propriedade rural ao abatedouro. Também foi considerado o sistema de terminação

\footnotetext{
${ }^{1}$ Recebido em 11 de outubro de 2014.

Aceito para publicação em 5 de março de 2015.

${ }^{2}$ Universidade Estadual de Montes Claros (Unimontes), Departamento de Ciências Agrárias, Av. Reinaldo Viana s/n, Bico da Pedra, Janaúba, MG 39440-000, Brasil. *Autor para correspondência: fredson.silva@ unimontes.br

${ }^{3}$ Departamento de Zootecnia, Escola de Veterinária, Universidade Federal de Minas Gerais (UFMG), Av. Antônio Carlos 6627, Cx. Postal 567, Belo Horizonte, MG 30123-970, Brasil. E-mail: flbtoral@ufmg.br
}

dos bovinos por meio da comunicação pessoal do técnico responsável pelo rebanho. Para entender o relacionamento das variáveis descritas, utilizaram-se a correlação dos componentes principais e as variáveis originais, os planos fatoriais, o círculo unitário, a análise de cluster e testes não-paramétricos. 0 escore de gordura subcutânea, a condição sexual, o peso da carcaça quente, o número de dentes e a propriedade rural, compuseram $68,26 \%$ da variação total. A conformação das carcaças e o sistema de terminação explicaram uma baixa parcela da variabilidade. As variáveis: propriedade rural (distância percorrida), número de dentes incisivos, sistema de terminação e escore de gordura subcutânea, influenciaram o número de carcaças com lesões. A condição sexual, o peso da carcaça quente e a conformação da carcaça não alteraram a proporção de carcaças com lesões.

TERMOS DE INDEXAÇÃO: Classificação de carcaças, bovinos inteiros, bovinos castrados, contusões, hematomas. 


\section{INTRODUÇÃO}

As exportações brasileiras de carne bovina em 2014 deverão alcançar aproximadamente 1,9 bilhão de toneladas em equivalente carcaça (CNA 2014). Apesar de o número expressivo, existem entraves, como quaisquer negócios, que devem ser conhecidos, um deles é a alta variabilidade quantitativa e qualitativa das características das carcaças.

Características como o peso, escore de gordura, conformação, cor da carne e da gordura, gordura intramuscular e declínio do pH são exemplos de parâmetros responsáveis por essa variabilidade. Esses parâmetros são associados ao animal, às condições de produção, manejo nas fazendas e aos processos que circundam o abate (Felício 2011). A aferição destas características nos matadouros-frigoríficos brasileiros, realizado a expensas do setor privado, regularizado pela Instrução Normativa no 9, de 4 de maio de 2004, traz os seguintes parâmetros obrigatórios: sexo, maturidade (número de dentes incisivos), peso da carcaça e acabamento da carcaça (Brasil 2004). Este detalhamento é importante para a composição do valor do produto.

Quando se levanta múltiplas variáveis, é possível que alguns destes parâmetros sejam redundantes, possibilitando seu descarte (Jolliffe 1972, Jolliffe 1973) e/ou atribuir peso ou importância diferentes de acordo com sua contribuição na variabilidade. Assim, intervenções nos componentes principais permitiriam melhores ajustes.

Como já dito, o manejo nas fazendas e o processo pré-abate são responsáveis, também, por algumas mudanças nas características das carcaças, dentre elas, o número de lesões (Huertas et al. 2010). Apesar de um período relativamente curto, as atividades são intensas e os animais, geralmente, às desconhecem. A infraestrutura, muitas vezes, também é deficiente (Grandin 2008). Conhecer quais vetores agem sobre as lesões pode direcionar as pesquisas e proporcionar manejos mais eficazes.

Diante do exposto, objetivou-se identificar quais os parâmetros das carcaças de bovinos anelorados são relevantes dentro do romaneio de abate e sua contribuição na variabilidade das características das carcaças. Além desse objetivo, determinar quais características dos animais e do manejo pré-abate estão relacionadas com o número de lesões nas carcaças.

\section{MATERIAL E MÉTODOS}

Utilizou-se um banco de dados com informações de 15.002 carcaças de bovinos anelorados dos anos de 2007 e 2008. Todos os animais foram abatidos em um matadouro-frigorífico com inspeção federal localizado em Janaúba/Minas Gerais/Brasil. As variáveis levantadas no romaneio de abate foram peso das carcaças quente $(n=15.002)$, conformação da carcaça $(n=4.175)$, escore de gordura subcutânea $(n=15.002)$, condição sexual $(n=15.002)$, número de dentes incisivos $(n=15.002)$, presença de lesões $(n=15.002)$ e distância percorrida da propriedade rural ao abatedouro $(n=15.002)$. Todas as informações foram reorganizadas de acordo com os objetivos dos pesquisadores. Levantou-se também o sistema de terminação $(n=7.683)$ dos bovinos por meio da comunicação pessoal do técnico responsável pelo rebanho.

Os animais, aleatoriamente incluídos na pesquisa, pertenciam a 32 municípios do Norte de Minas Gerais. Os municípios e suas respectivas participações em percentagem foram: Janaúba
$(17,77)$, Verdelândia $(3,90)$, Pai Pedro $(0,13)$, Jaíba $(10,07)$, Capitão Enéas $(7,75)$, Francisco Sá $(4,43)$, Matias Cardoso $(1,56)$, Itacarambi $(14,38)$, Espinosa $(0,57)$, Montes Claros $(9,99)$, Juramento $(0,25)$, Manga $(0,49)$, Mirabela $(0,26)$, Januária $(0,14)$, Claro dos Poções $(0,42)$, São João da Lagoa $(0,29)$, Engenheiro Navarro $(0,50)$, Coração de Jesus $(0,29)$, Salinas $(0,29)$, Patis $(1,52)$, Brasília de Minas $(1,71)$, Jequitaí $(6,00)$, São João do Pacuí $(0,43)$, Francisco Dumont $(1,36)$, Rubelita $(0,69)$, São João da Ponte $(8,55)$, Joaquim Felício $(0,49)$, Pedras de Maria da Cruz $(0,25)$, Varzelândia $(0,22)$, São Francisco $(3,95)$, Ponto Chique $(0,14)$ e Brasilândia de Minas $(1,22)$.

Para avaliação da condição sexual, as carcaças foram classificadas de acordo com a Instrução Normativa no 37, de 29 de dezembro de 2004 do Ministério da Agricultura, Pecuária e Abastecimento (Brasil 2004). A normativa brasileira estabelece a divisão em quatro condições sexuais, que são: macho não-castrado, macho castrado, novilha, vaca de descarte (oito dentes incisivos permanentes).

0 peso das carcaças quentes foi obtido por meio da soma das meias-carcaças medidas na linha J da Linha de Inspeção. 0 número de dentes incisivos de cada animal foi determinado pela leitura da arcada dentária na linha C.

O escore de gordura subcutânea e conformação das carcaças foram determinados visualmente pelo colaborador do matadouro-frigorífico treinado para a avaliação dessas variáveis. 0 escore de gordura seguiu a escala de classificação de carcaças de 1 a 5 (Felício 2011), em que 1 corresponde a ausência de gordura (0$1 \mathrm{~mm}) ; 2=$ escassa $(1-3 \mathrm{~mm}) ; 3=$ mediana $(3-6 \mathrm{~mm}) ; 4=$ uniforme $(6-10 \mathrm{~mm})$ e 5 = excessiva $(>10 \mathrm{~mm})$. A conformação foi determinada pela escala de classificação de carcaças de 1 a 3 , sendo que 1 corresponde a conformação inferior (subcôncavo e côncavo); 2 = boa (retilíneo) e 3 = excelente (subconvexo e convexo). Ambas as medidas foram obtidas na linha J.

Para estimativa da distância rodoviária percorrida das fazendas ao abatedouro foi utilizado o Google Maps- ${ }^{\circledR} 2013$ Google.

As carcaças dos animais foram classificadas de acordo com o sistema de terminação em: bovinos confinados ou criados em pasto.

A análise multivariada foi aplicada para se entender o relacionamento entre as variáveis descritas nos romaneios de abate de bovinos. Para isso, utilizou-se a correlação dos componentes principais e as variáveis originais, os planos fatoriais, o círculo unitário, a análise de cluster e testes não-paramétricos $(\mathrm{P}<0,05)$.

Depois de realizado o agrupamento das variáveis, utilizou-se a análise de componentes principais para identificar quais as variáveis foram relevantes dentro do romaneio de abate e sua contribuição na variabilidade, conforme recomendações de Jolliffe (1973). Este método estipula que o número de variáveis descartadas deve ser igual ao número de componentes que a variância (autovalor) seja menor a 0,7.

Para avaliação dos vetores que influenciaram a variável suplementar "lesões", encontrados no círculo unitário, utilizou-se a análise de cluster. Para a formação de grupos nos dendogramas, utilizou-se o método de encadeamento único (single linkage) que se baseia na distância mínima, utilizando a regra do vizinho mais próximo.

Realizou-se também teste não paramétrico Mann Whitney ou Kruskal-Wallis $(\mathrm{P}<0,05)$, de acordo com o número de grupos obtidos na análise de Cluster, para confrontar as frequências das variáveis.

\section{RESULTADOS}

Os componentes principais, autovalores e porcentagem da variância explicada pelos romaneios estão no Quadro 1. 
Quadro1. Componentes principais, autovalores $(\Lambda)$ e porcentagem da variância explicada pelos componentes (VCP) nos romaneios de carcaças bovinas

\begin{tabular}{lccccccc}
\hline \multicolumn{1}{c}{ Variável } & \multicolumn{7}{c}{ Cargas dos componentes principais } \\
\cline { 2 - 8 } & Fator 1 & Fator 2 & Fator 3 & Fator 4 & Fator 5 & Fator 6 & Fator 7 \\
\hline Condição sexual & $\mathbf{- 0 , 8 2}$ & $-0,20$ & $-0,26$ & 0,20 & $-0,01$ & 0,15 & $-0,41$ \\
Peso & $\mathbf{- 0 , 7 9}$ & 0,18 & 0,27 & 0,00 & $-0,01$ & 0,48 & 0,24 \\
Número de dentes & $\mathbf{0 , 7 3}$ & 0,21 & 0,15 & 0,15 & 0,51 & 0,31 & $-0,15$ \\
Conformação & $-0,60$ & 0,29 & 0,20 & $-0,57$ & 0,38 & $-0,22$ & $-0,08$ \\
Escore gordura & 0,20 & $\mathbf{0 , 8 6}$ & 0,17 & $-0,05$ & $-0,39$ & 0,03 & $-0,18$ \\
Propriedade rural & 0,25 & 0,06 & $\mathbf{- 0 , 8 0}$ & $-0,48$ & $-0,04$ & 0,25 & 0,03 \\
Sistema Terminaçãoa & 0,34 & $-0,55$ & 0,52 & $-0,44$ & $-0,22$ & 0,19 & $-0,15$ \\
Autovalores $(K)$ & 2,3834 & 1,2502 & 1,1443 & 0,8187 & 0,6081 & 0,4892 & 0,3061 \\
VCP $_{\text {VCP acumulada }}^{34,05}$ & 17,86 & 16,35 & 11,70 & 8,69 & 6,99 & 4,37 \\
& 34,05 & 51,91 & 68,26 & 79,96 & 88,65 & 95,64 & 100,00
\end{tabular}

Valores em destaque possuíram significância igual ou maior que $0,70 .{ }^{a}$ Comunicação dos técnicos que acompanharam os abates.

Quadro 2. Coeficiente de correlação simples $(P<0,05)$ de variáveis levantadas romaneios de carcaças bovinas e os componentes principais

\begin{tabular}{lccccccc}
\hline & Fator 1 & Fator 2 & Fator 3 & Fator 4 & Fator 5 & Fator 6 & Fator 7 \\
\hline Propriedade rural & 0,25 & 0,06 & $-0,80$ & $-0,48$ & ns $^{\mathrm{a}}$ & 0,25 & ns \\
Condição sexual & $-0,82$ & $-0,20$ & $-0,26$ & 0,20 & ns & 0,15 & $-0,41$ \\
Peso & $-0,78$ & 0,18 & 0,27 & ns & ns & 0,48 & 0,24 \\
Número de dentes & 0,73 & 0,21 & 0,15 & 0,15 & 0,51 & 0,31 & $-0,15$ \\
Conformação & $-0,60$ & 0,29 & 0,20 & $-0,57$ & 0,38 & $-0,22$ & $-0,08$ \\
Escore gordura & 0,20 & 0,86 & 0,17 & $-0,05$ & $-0,39$ & ns & $-0,18$ \\
Sistema Terminação & 0,34 & $-0,55$ & 0,52 & $-0,44$ & $-0,22$ & 0,19 & $-0,15$
\end{tabular}

Nota-se que até o fator 3, explica-se $68,26 \%$ da variância total, sendo que a condição sexual, peso das carcaças quentes, número de dentes incisivos, escore de gordura subcutânea e a propriedade rural, a compõe. A conformação da carcaça e o sistema de terminação não foram significativos para a composição da variação.

A conformação da carcaça e os fatores 1 e 3 apresentou correlação alta (Quadro 2), conforme critério de Cohen (1988). 0 sistema de terminação também apresentou correlação alta com os fatores 2 e 3.

Na Figura 1 está apresentada a projeção espacial da ordenação dos vetores. Complementando o resultado do Quadro 1 , o escore de gordura subcutânea está mais próximo do círculo unitário, portanto, possuiu maior contribuição com a variação total. A condição sexual, peso das carcaças e número de dentes, foram os demais vetores que estão próximos ao círculo, nessa ordem de contribuição para a variação.

Na mesma figura colocou-se o vetor lesão como uma variável suplementar. As variáveis, propriedade rural, número de dentes incisivos, sistema de terminação e escore de gordura subcutânea, que ficaram nos quadrantes I e IV, influenciaram o número de lesões, pois estavam na sua mesma posição no círculo unitário. Já a condição sexual, peso das carcaças quentes e conformação das carcaças ficaram no lado oposto, portanto, não influenciaram as lesões.

Como nem todas as variáveis analisadas apresentaram influência sob o número de carcaças com lesões, avaliaram-se somente as que contribuíram com essa característica.

$\mathrm{Na}$ análise do dendograma, apresentado na Figura 2,

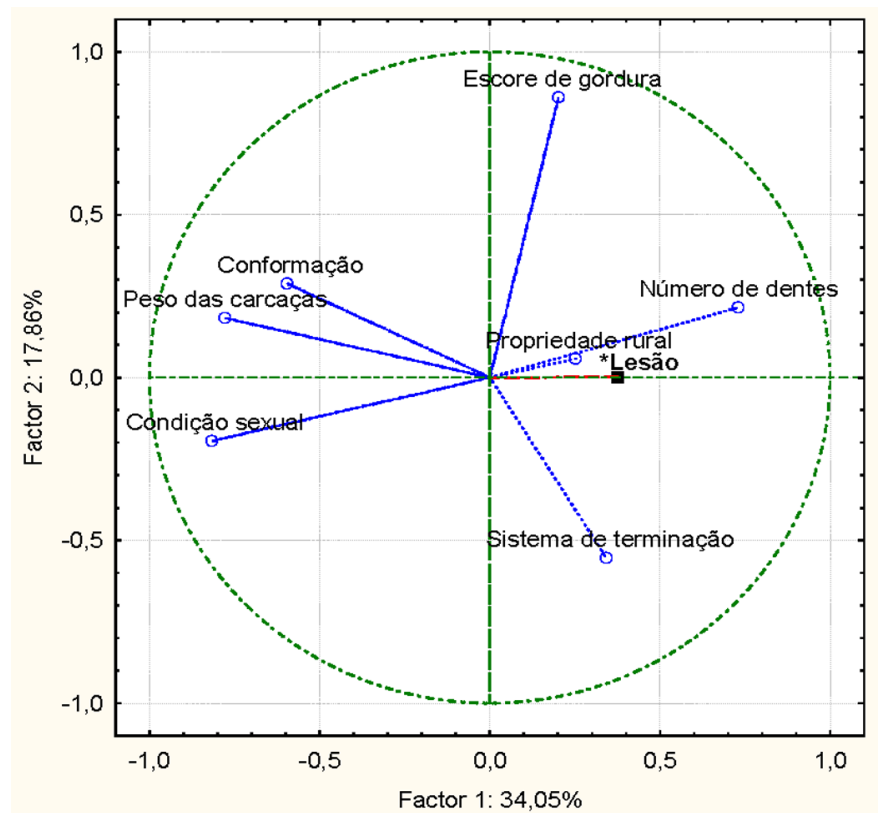

Fig.1. Projeção espacial da ordenação dos vetores das variáveis levantadas nos romaneios das carcaças nos dois componentes principais. ? Variável suplementar.

houve formação de dois grupos, sendo que os bovinos que foram transportados em uma maior distância, a partir de $240 \mathrm{~km}$, apresentaram mais lesões em suas carcaças $(7,25$ vs. $11,97 \%, \mathrm{P}=0,0002)$.

Avaliando o dendograma na Figura 3, nota-se que foram formados 4 grupos, animais com menos de 2 dentes incisi- 


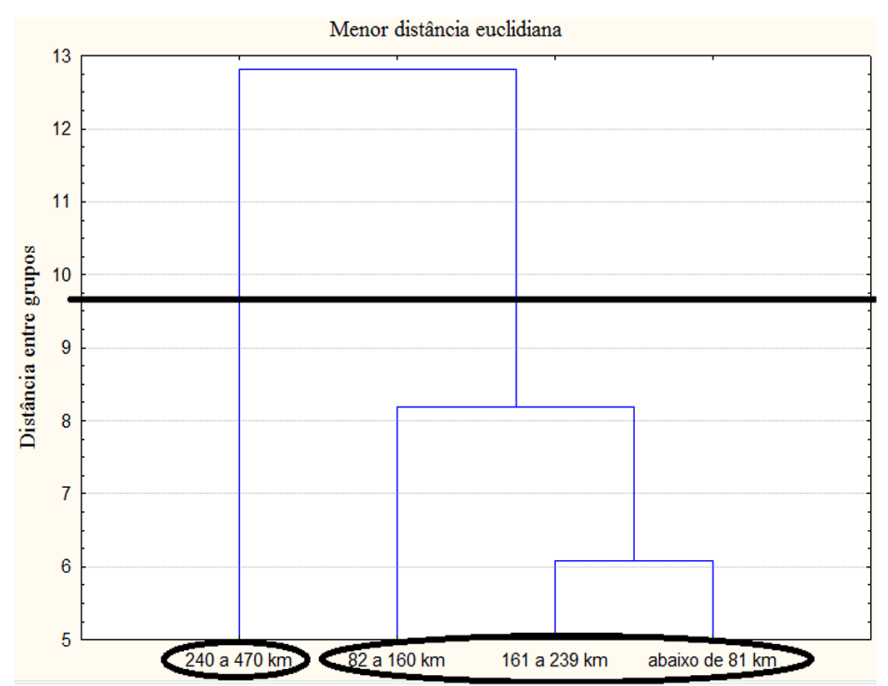

Fig.2. Dendograma da análise de cluster da distância (km) da propriedade rural ao abatedouro e lesão de bovinos.

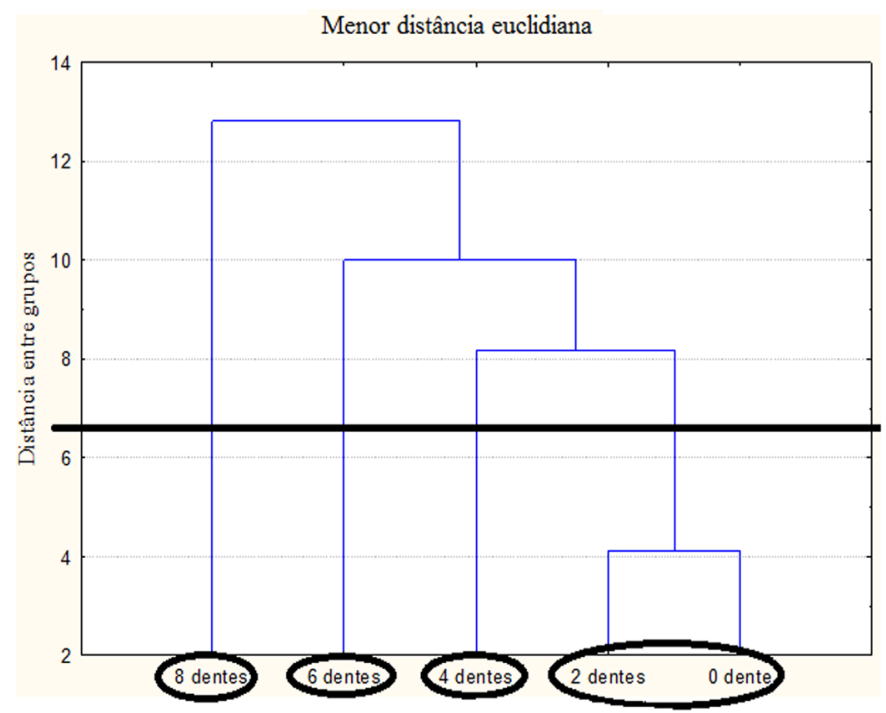

Fig.3. Dendograma da análise de cluster do número de dentes e lesão de bovinos.

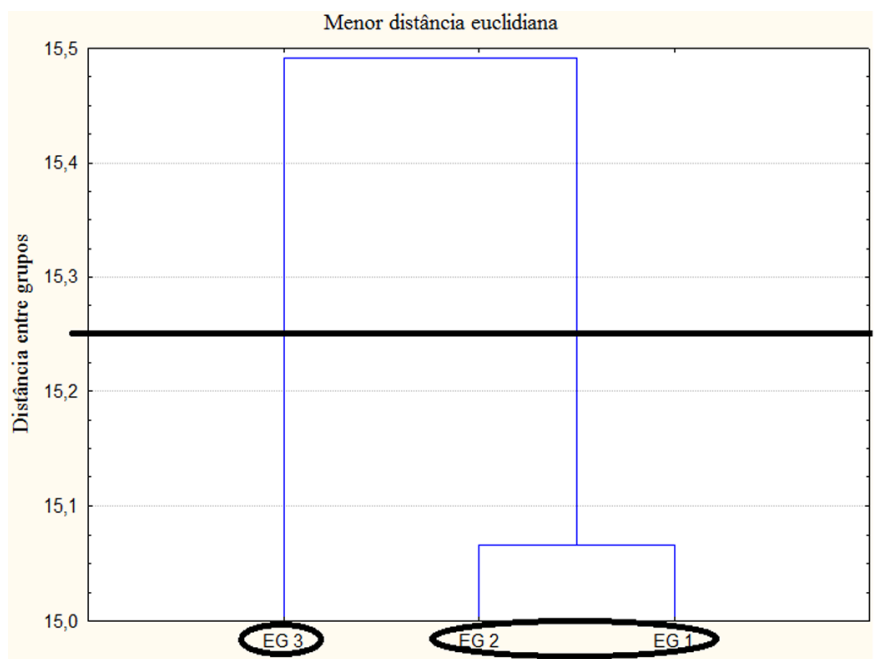

Fig.4. Dendograma da análise de cluster do escore de gordura (EG) e lesão de bovinos. vos, 4 dentes, 6 dentes e 8 dentes. A porcentagem de lesões aumentou diretamente com o acréscimo de dentes incisi$\operatorname{vos}(5,14 ; 7,45 ; 8,43 ; 11,98 \%, \mathrm{P}<0,0001)$.

A porcentagem de lesões aumentou quando os animais foram confinados $(8,52 ; 11,77 \%, \mathrm{P}=0152)$. Quando se avaliou o escore de gordura subcutânea, houve a formação de dois grupos, carcaças com maiores escores (EG 3) tiveram mais lesões do que as carcaças que possuíam menores (EG 1 e 2) (Fig.4).

\section{DISCUSSÃO}

Nos matadouros-frigoríficos brasileiros que possuem o Serviço de Inspeção Federal é comum a utilização de parâmetros para compor o preço da arroba bovina e/ou situações de bonificação e penalização das carcaças. Diante do exposto, melhores bonificações dos componentes principais podem melhorar as carcaças, pois podem diminuir a variabilidade.

Além da atenção às características intrínsecas das carcaças, pecuaristas e/ou proprietários de matadouros-frigoríficos podem investir em auxílio técnico nas propriedades rurais, pois essa variável responde por uma alta porcentagem da variação total.

A conformação das carcaças pode ser descartada, conforme critérios de Jolliffe $(1972,1973)$, ou seja, as carcaças não necessitam receber escore dessa variável. O levantamento do sistema de terminação, neste caso realizado por comunicação pessoal, também pode ser descartado. Caso apresentassem valores significativos para as cargas dos componentes principais, haveria redundâncias, devido à alta correlação com os fatores 1, 2 ou 3.

Quando se avalia a conformação, carcaças bovinas de animais Bos indicus geralmente são classificadas como retilíneas, em virtude da menor deposição de músculo em comparação a animais Bos taurus (Silva et al. 2008), o que resulta em pequena variação.

Além das questões inerentes aos manejos nutricional e sanitário e às variações genotípicas, o manejo no momento pré-abate, incluindo aqui embarque, transporte e desembarque, pode afetar as características quantitativas e qualitativas das carcaças e carnes (Petherick \& Phillips 2009; Bertoloni et al. 2012; Romero et al. 2013). As lesões nas carcaças diminuem o rendimento da carcaça devido à extirpação do local afetado (Ferguson \& Warner 2008), além disso, o valor pago por quilo de carcaça também pode reduzir de acordo com a tipificação utilizada pelo matadouro-frigorífico.

Diferente deste ensaio, Jarvis et al. (1995) relataram influência da condição sexual no número de lesões de carcaças bovinas e, segundo Voisinet et al. (1997), novilhas são mais reativas do que os novilhos. Já Restle et al. (1994), corroborando com este trabalho, não verificaram problemas com agressividade e sodomia dos inteiros sobre os castrados. Nesta pesquisa, devido à alta correlação da condição sexual e do peso das carcaças quentes, não houve influência desse último; ambos explicaram o fator 1 .

Como neste trabalho, González et al. (2012) mostraram que transportes com maior duração podem diminuir o bem-estar dos animais e, segundo Andrade et al. (2008), 
aumentar o número de lesões na carcaça. Portanto, as variáveis envolvidas nos transportes devem receber atenção especial, sobretudo em jornadas longas. Como exemplo, segundo Petherick \& Phillips (2009), a área disponível por animal durante o transporte, se mal delimitada, pode causar uma série de eventos potencialmente traumáticos aos animais. Já para Hoffman \& Lühl (2012), ao contrário deste experimento, a distância percorrida, em um intervalo de duração de 3 a 8 horas, teve efeito mínimo para o aparecimento de lesões em bovinos.

Como bovinos com o maior número de dentes incisivos são mais velhos, respeitando as variações individuais e entre genótipos (Lawrence \& Fowler 2002), o número de carcaças com lesões aumentou diretamente com a idade. Portanto, abater animais com menor idade é, utilizando esse parâmetro, uma vantagem para o produtor rural, pois terão menor extirpação de carne. Vantagens também são atribuídas ao abatedouro, pois terão menos cortes desfigurados causados por lesões.

Quando se avalia o resultado do sistema de terminação e lesão, possivelmente, a infraestrutura dos confinamentos, nesta região mineira, necessite de melhorias. Como descrito por Garcia et al. (2008), apresentando a auditória que quantifica e qualifica a carne bovina dos Estados Unidos, houve diminuição das lesões nas carcaças devido ao investimento da indústria no manejo dos bovinos. A correlação de Pearson entre o sistema de terminação e o escore de gordura subcutânea foi alta, ou seja, bovinos terminados em confinamento apresentaram maior escore de gordura, assim o resultado e a discussão desse parágrafo servem para os dois casos. Provavelmente, o que determinou o maior número de lesões nas carcaças com maior escore de gordura de cobertura foi a infraestrutura do confinamento.

\section{CONCLUSÕES}

O escore de gordura subcutânea, a condição sexual, o peso da carcaça quente e o número de dentes incisivos determinam a maior parte da variabilidade das carcaças de bovinos anelorados.

A conformação da carcaça e o sistema de terminação (pasto ou confinado) não contribuem com a variabilidade das características das carcaças.

O número de lesões nas carcaças foi maior em animais que foram transportados por um maior período (distância percorrida acima de $240 \mathrm{~km}$ ), em animais com maior número de dentes incisivos e em animais terminados em confinamento.

Agradecimentos.- À Fundação de Amparo à Pesquisa do Estado de Minas Gerais (FAPEMIG), pelo auxílio financeiro para publicação deste artigo.

\section{REFERÊNCIAS}

Andrade E.N., Roça R.O., Silva R.A.M.S., Gonçalves H.C. \& Pinheiro R.S.B. 2008. Prevalência de lesões em carcaças de bovinos de corte abatidos no Pantanal Sul Mato-Grossense transportados por vias fluviais. Ciênc. Tecnol. Aliment. 28(4):822-829.

Bertoloni W., Silva J.L., Abreu J.S. \& Andreolla D.L. 2012. Bem-estar e taxa de hematomas de bovinos transportados em diferentes distâncias e modelos de carroceria no estado do Mato Grosso, Brasil. Revta Bras. Saúde Prod. Anim. 13(3):850-859.

Brasil 2004. Sistema Brasileiro de Classificação de Carcaças de Bovinos. Instrução Normativa no 09/2004, Ministério da Agricultura, Pecuária e Abastecimento (MAPA). Disponível em <http://extranet.agricultura. gov.br/sislegis/action/detalhaAto.do?method=consultarLegislacaoFed eral> Acesso em 30 mar. 2014.

Cohen J. 1988. Statistical Power Analysis for the Behavioral Sciences. Erlbaum, Hills Dale, NJ.

Confederação Nacional da Agricultura 2014. Balanço da Pecuária Bovídea de Corte. Fórum Nacional Permanente da Pecuária de Corte. Disponível em <http://www.canaldoprodutor.com.br/>

Felício P.E. 2011. Classificação, tipificação e qualidade da carne bovina. VI Congresso Brasileiro de Ciência e Tecnologia de Carnes e publicado nos Anais de Palestras, São Pedro, SP, p.127-133. (Palestra)

Ferguson D.M. \& Warner R.D. 2008. Have we under estimated the impact of pre-slaughter stress on meat quality in ruminants?: a review. Meat Sci. 80:12-19.

Garcia L.G., Nicholson K.L., Hoffman T.W., Lawrence T.E., Hale D.S., Griffin D.B., Savell J.W., VanOverbeke D.L., Morgan J.B., Belk K.E., Field T.G., Scanga J.A., Tatum J.D. \& Smith G.C. 2008. National Beef Quality Audit-2005: survey of targeted cattle and carcass characteristics related to quality, quantity, and value of fed steers and heifers. J. Anim. Sci. 86:3533-3543.

González L.A., Schwartzkopf-Genswein K.S., Bryan M., Silasi R. \& Brown F. 2012. Benchmarking study of industry practices during commercial long haul transport of cattle in Alberta, Canada. J. Anim. Sci. 90:3606-3617.

Grandin T. 2008. Engineering and design of holding yards, loading ramps, and handling facilities for land and sea transport of livestock. Veterinaria Italiana 44:235-245.

Hoffman L.C. \& Lühl J. 2012. Causes of cattle bruising during handling and transport in Namibia. Meat Sci. 92:115-124.

Huertas S.M., Gil A.D., Piaggio J.M. \& Van Eerdenburg F.J.C.M. 2010. Transportation of beef cattle to slaughter houses and how this relates to animal welfare and carcase bruising in an extensive production system. Animal Welfare 19:281-285.

Jarvis A.M., Selkirk L. \& Cockram M.S. 1995. The influence of source, sex class and pre-slaughter handling on the bruising of cattle at two slaughter houses. Livest. Prod. Sci. 43:215-224.

Jolliffe I.T. 1972. Discarding variables in a principal component analysis. I. Artificial data. Appl. Stat. 21:160-173.

Jolliffe I.T. 1973. Discarding variables in a principal component analysis. II. Real data. Appl. Stat. 22:21-31.

Lawrence T.L.J. \& Fowler V.R. 2002. Growth of Farm Animals. 2nd ed. CABI Publishing, London. 347p.

Petherick J.C. \& Phillips C.J.C. 2009. Space allowances for confined livestock and their determination form allometric principles. Appl. Anim. Behav. Sci. 117:1-12.

Restle J., Grassi C. \& Feijó G.L.D. 1994. Evolução de peso de bovinos de corte inteiros ou castrados em diferentes idades. Pesq. Agropec. Bras. 29:1631-1635.

Romero M.H., Uribe-Velásquez L.F., Sánchez J.A. \& Miranda-de La Lama G.C. 2013. Risk fator sinfluencing bruising and high muscle $\mathrm{pH}$ in Colombian cattle carcasses due to transport and pre-slaughter operations. Meat Sci. 95:256-263.

Silva F.V., Rocha Júnior V.R., Barros R.C., Pires D.A.A., Menezes G.C.C. \& Caldeira L.A. 2008. Ganho de peso e características de carcaça de bovinos Nelore castrados ou não-castrados terminados em confinamento. Revta Bras. Zootec. 37:2199-2205.

Voisinet B.D., Grandin T., Tatum J.D., O'Connor S.F. \& Struthers J.J. 1997. Feedlot cattle with calm temperaments have higher average daily gains than cattle with excitable temperaments. J. Anim. Sci. 75:892-896. 